\title{
Análisis para determinar la edad óptima para el manejo quirúrgico del pie equino en pacientes con parálisis cerebral infantil
}

\author{
Analysis to determine optimal age for surgical management of \\ equinus foot in patients with childhood cerebral palsy \\ Gaytán-Fernández S, ${ }^{*, \neq}$ Chaidez P, ${ }^{\ddagger}$ García-Galicia A, ${ }^{*}$ Martínez-Asención P,*
Barragán-Hervella RG, ${ }^{*}$ Corpus-Mariscal E, ${ }^{*}$ Jiménez-Reyes M,*,§ Montiel-Jarquín AJ*
}

Hospital Shriners para Niños de la Ciudad de México.

RESUMEN. Introducción: La parálisis cerebral infantil es una lesión cerebral no progresiva que ocurre antes, durante o después del parto y provoca daño neurológico variable que oscila de leve hasta discapacitante. La deformidad en el pie equino se trata conservadoramente en edades tempranas, pero ¿cuándo está indicado el manejo quirúrgico? Objetivo: Determinar la edad óptima para el manejo quirúrgico del pie equino en pacientes con PCI. Material y métodos: Estudio retrospectivo realizado en pacientes con diagnóstico de PCI (todos los tipos), tratados quirúrgicamente con alargamiento del tendón de Aquiles abierto o percutáneo y valorados con notas de la consulta externa; los pacientes tuvieron de 1-16 años y un seguimiento promedio de seis años, valorándose la marcha con recidiva de deformidad y la marcha plantígrada. Resultados: Se analizaron 55 pacientes, 74 con pie equino (29 niñas y 45 niños); de éstos, presentaron más recidivas los operados antes de los seis años de edad, con un período vulnerable entre los cuatro y seis años. La monoplejía registró 100\% de recidivas, mientras que la triplejía $0 \%$. La cirugía abierta se asoció con recurrencia en 50\% y la cirugía percutánea sólo en 19\%.
ABSTRACT. Introduction: Childhood cerebral palsy, a non-progressive brain injury, occurs before, during or after delivery, with variable neurological damage from mild to disabling. The deformity in equine is treated conservatively at an early age, but when is surgical management indicated? Objective: Our goal was to determine the optimal age for surgical management of the equine foot in CCP patients. Material and methods: Retrospective study, in patients diagnosed with CCP (all types), treated surgically with open or percutaneous Achilles tendon elongation, assessed with external consultation notes, in patients aged 1-16 years, and average follow-up of 6 years, evaluating progress with relapse of deformity and gait with plantigrade support. Results: 55 patients, 74 equinus feet (29 in girls, 45 in boys) were analyzed with surgical treatment. Those treated before six years old presented relapses, with vulnerable period in 4-6 years. Monoplegia presented 100\% relapses, and triplegia presented $0 \%$. Open surgery presented $50 \%$ recurrence and percutaneous technique only $19 \%$. Conclusion: In our institution, the optimal age is suggested in 6-12 years. Percutaneous technique over the open,

\section{Nivel de evidencia: IV}

* Unidad Médica de Alta Especialidad del Hospital de Traumatología y Ortopedia de Puebla, Centro Médico Nacional «Gral. de Div. Manuel Ávila Camacho» del Instituto Mexicano del Seguro Social. Puebla, Puebla.

${ }^{\ddagger}$ Hospital Shriners para Niños. Ciudad de México.

$\S_{\S}^{\S}$ Facultad de Medicina de la Universidad Popular Autónoma del Estado de Puebla. Puebla, Puebla.

Dirección para correspondencia:

Arturo García-Galicia

Jefatura de División de Investigación en Salud, Unidad Médica de Alta Especialidad del Hospital de Traumatología y

Ortopedia de Puebla, «Gral. de Div. Manuel Ávila Camacho» del Instituto Mexicano del Seguro Social.

Diagonal Defensores de la República, esq. 6 poniente s/n, Col. Amor, C.P. 72140, Puebla, Puebla.

Teléfono: +52 2222493099 ext. 208

Móvil: 2221945360

E-mail: arturo.garciaga@imss.gob.mx 
Conclusiones: En nuestra institución, se sugiere que la edad óptima de la cirugía esté entre los 6-12 años. Es preferible la técnica percutánea sobre la abierta, debiendo poner mayor atención en el seguimiento de la monoplejía.

Palabras clave: Marcha, equino, pie, cirugía, edad, resultados.

\section{Introducción}

La parálisis cerebral infantil (PCI) se define como una lesión no progresiva y motriz del cerebro, la cual ocurre antes, durante o después del parto. Este compromiso neurológico es variable y provoca desde daño leve hasta casos severos con discapacidad total y permanente. A pesar de estar establecido que la lesión neurológica es estática, no lo es a nivel del sistema musculoesquelético, ya que presenta cambios significativos y progresivos esencialmente en la función motriz al traer como consecuencia cambios en los huesos, articulaciones y músculos. Dentro de los problemas musculares más frecuentes en la PCI espástica se destaca la deformidad del pie equino, la cual se puede tratar de manera conservadora en edades tempranas. ${ }^{1}$ Sin embargo, durante el crecimiento del paciente, la deformidad tiende a recurrir, ya que la tibia crece longitudinalmente más rápido que el tríceps sural. De esta manera, la cirugía está indicada, especialmente ante el fracaso del tratamiento conservador, o bien, cuando la deformidad es muy grave.

Es controversial determinar la edad a la que se debe iniciar el manejo quirúrgico, principalmente para evitar recidivas; ${ }^{1,2}$ sin embargo, se puede tomar como guía la velocidad del crecimiento muscular proporcional al crecimiento óseo. El músculo duplica su longitud entre el nacimiento y los cuatro años de edad y, así, la longitud vuelve a duplicarse entre los cuatro años y la adultez. Esto explica por qué los niños a los que se les realizan alargamientos antes de los cuatro años de edad requieren un segundo alargamiento a causa de las recidivas de la deformidad, mientras que el alargamiento realizado should be preferred, and greater attention should be paid to monitoring monoplexy.

Keywords: Gait, equinus, foot, surgery, age, results. después de los cuatro años de edad sirve para toda la vida. ${ }^{3,4}$

El objetivo de este estudio fue revisar los resultados del alargamiento del tendón de Aquiles en pacientes con PCI tratados a partir de los seis meses hasta los 20 años de edad, determinando así la edad como factor pronóstico de recidiva en nuestra población.

\section{Material y métodos}

A través del expediente clínico, se revisaron 55 pacientes y en total 74 pies en equino, los cuales fueron tratados quirúrgicamente; de éstos 29 fueron niñas y 45 niños. También, de manera retrospectiva, se revisó de estos pacientes el diagnóstico de PCI del tipo monoplejía, diplejía, triplejía, paraplejía y cuadriplejía, que tuvieran equino del tobillo, y hubieran sido tratados de manera quirúrgica con alargamiento del tendón de Aquiles de manera abierta (tipo Hauser $y$ White) o percutánea (por deslizamiento), antes y después de los seis años de edad.

Todos los alargamientos produjeron que se redujera completamente el pie equino. Estos pacientes permanecieron en el postoperatorio por seis semanas con una férula de fibra de vidrio muslo-podálica. Posterior a su retiro, se manejó órtesis tobillo-pie y fisioterapia (consistente en ejercicios de estiramiento de tríceps sural y entrenamiento de marcha). Se valoraron los resultados de acuerdo con las notas de seguimiento en la consulta externa; la edad osciló de uno a 16 años, con un promedio de seis años de seguimiento, determinando durante la marcha la presencia de equino recidivante o persistencia del pie plantígrado.

\section{Tabla 1: Relación entre el tipo de PCI y el manejo quirúrgico junto con el número de recidivas.}

\begin{tabular}{|c|c|c|c|c|c|c|}
\hline Tipo de PCI & Cirugía abierta & Cirugía cerrada & Recidivas & $\begin{array}{l}\text { Porcentaje } \\
\text { de recidivas }\end{array}$ & Sin recidivas & Total \\
\hline Hemiplejía & 2 & 8 & 6 & 60 & 4 & 10 \\
\hline Diplejía & 8 & 17 & 4 & 16 & 21 & 25 \\
\hline Paraplejía & 7 & 15 & 8 & 57 & 14 & 22 \\
\hline Triplejía & 1 & 4 & 0 & 0 & 5 & 5 \\
\hline Monoplejía & 2 & 0 & 2 & 100 & 0 & 2 \\
\hline Cuadriplejía & 2 & 8 & 1 & 10 & 9 & 10 \\
\hline Total & 22 & 52 & 21 & & 53 & 74 \\
\hline
\end{tabular}




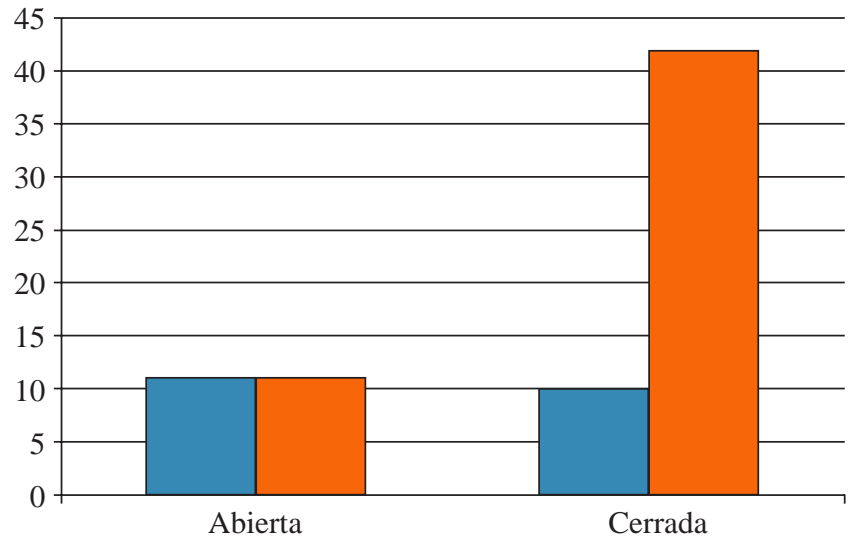

Tipo de cirugía

Recidivas

Sin recidivas

Figura 1: Relación entre tipo de cirugía y presencia de recidivas.

\section{Resultados}

Se analizó el resultado clínico de los 74 pies en equino tratados quirúrgicamente. En la Tabla 1 se muestra el tipo de PCI, la técnica quirúrgica utilizada y el porcentaje de recidivas. Destaca que el tipo de PCI monopléjico registró una recidiva de $100 \%$ (dos pacientes, ambos manejados con cirugía abierta) y que la triplejía registró cero recidivas (cinco pacientes, uno con técnica abierta y cuatro con técnica cerrada). En todos los tipos de PCI predominó la técnica cerrada, salvo en el caso mencionado de monoplejía. En cuanto al tipo de técnica quirúrgica, la comparación gráfica de las recidivas se muestra en la Figura 1, con hasta 50\% de recidivas en la técnica abierta y 19\% en la cirugía cerrada.

En los tipos de PCI con mayor número de pacientes, las recidivas registradas variaron desde 16 hasta $60 \%$. El contraste entre porcentaje de recidivas y tipo de PCI se hace evidente en la Figura 2.

Por otra parte, en cuanto al momento de la cirugía, la mayoría de los pacientes presentan alguna recidiva cuando fueron tratados antes de los seis años, lo que marca la desviación en un período vulnerable entre los cuatro y seis años de edad, tal como se ilustra en la Figura 3.

La ausencia de recidivas es predominante cuando la cirugía correctiva se realiza después de los seis años, teniendo como moda los 12 años, tal como se hace evidente en la Figura 4.

\section{Discusión}

El presente estudio se basó en la velocidad del crecimiento muscular, el cual es proporcional al crecimiento óseo. Los hallazgos evidencian la necesidad de realizar una revisión y de reinterpretar la literatura, pues sugieren que el practicar el alargamiento quirúrgico en pacientes con PCI a temprana edad (antes de los seis años) — tal como ocurrió en nuestra institución- mostró un el alto índice de recidivas y la subsecuente necesidad de realizar una segunda intervención de revisión.

Después de los seis años pueden crearse las condiciones óptimas para el manejo quirúrgico sin riesgo de la reaparición de la deformidad, teniendo una moda de 12 años, lo que permitiría establecer como rango óptimo para la intervención quirúrgica la edad de seis a 12 años.

Además, la frecuencia de recidivas parece asociarse con el tipo de PCI, pues se presentaron la monoplejía en $100 \%$ de ellas y $0 \%$ de recidivas para la triplejía.

Destaca la observación de que la cirugía abierta presentó 50\% de recidivas, mientras que la cirugía cerrada apenas 19\%. Dichos resultados requieren ser confrontados con estudios anteriores como los de Rattey y colaboradores, ${ }^{2}$ quienes, con una muestra similar de pacientes con PCI, pero sólo con hemiplejía y diplejía con equino realizaron un alargamiento abierto «en Z», con un seguimiento aproximado de 10 años y determinaron que con este manejo en niños $\geq 6$ años de edad no se presentaron recidivas de la deformidad, pero no así con los niños ope-

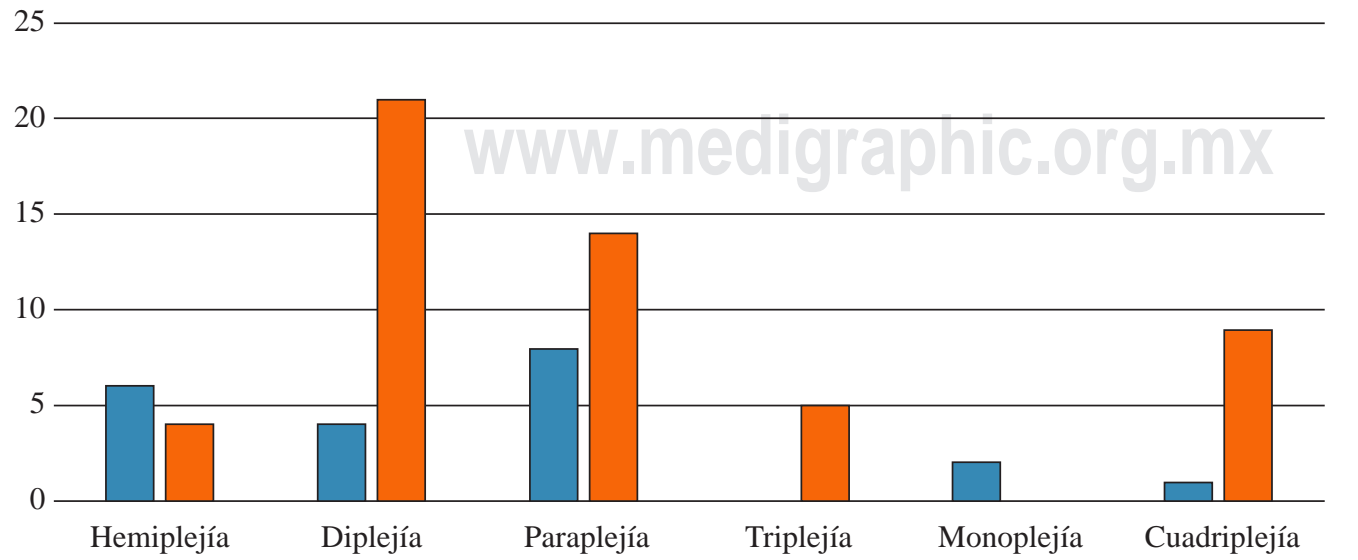

$\square$ Con recidivas

Sin recidivas
Figura 2:

Pacientes con recidivas y sin recidivas de acuerdo con el tipo de PCI. 
rados antes de cumplir los cuatro años. También asociaron los índices de recurrencia a los pacientes dipléjicos en $18 \%$ y en $41 \%$ a hemipléjicos. ${ }^{5}$

En el presente trabajo se laboró de manera amplia con los pacientes con PCI, incluyendo todos sus tipos; asimismo se valoraron los dos manejos quirúrgicos, lo cual es nuevo en el reporte de estudios relacionados con el tema.

Por otro lado, Koman y Smith evaluaron 31 estudios, de los cuales nueve reportaron que, en menores de siete años de edad, el tratamiento quirúrgico tenía un riesgo más alto de recurrencias que el realizado en niños mayores a esta edad. A su vez encontraron índices menores de recurrencia de la deformidad en pacientes con diplejía comparados con pacientes con hemiplejía. ${ }^{4}$

Mención aparte merece el estudio de Dietz, quien utilizó el alargamiento del tendón de Aquiles en equino del tobillo en PCI, recomendando alternativamente alargamientos de la fascia de gastrocnemios o de tendón de Aquiles únicamente a los pacientes que presenten equino espástico durante la marcha. $^{3}$

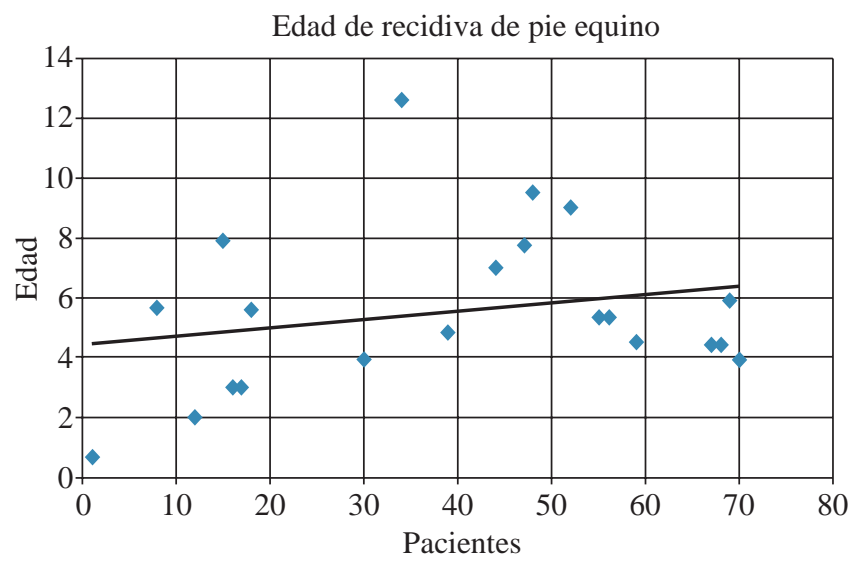

Figura 3: Predominio de recidiva de pie equino en pacientes menores de 6 años.

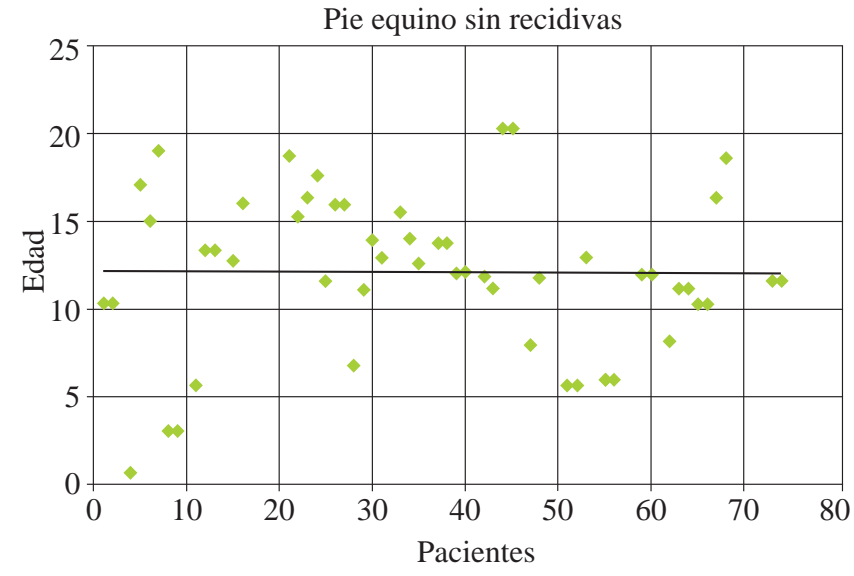

Figura 4: Pacientes mayores de 6 años sin recidivas.

\section{Conclusión}

Estos resultados permiten sugerir en nuestra institución, que el manejo quirúrgico del pie equino en el paciente con PCI sea en el rango de edad de seis a 12 años, considerando mayor atención en el manejo de los pacientes con monoplejía, ya que presenta más recidivas; se debe preferir la técnica percutánea sobre la cirugía abierta debido a que registra menos recurrencias.

Bibliografía

1. Koman LA, Smith BP, Barron R. Recurrence of equinus foot deformity in cerebral palsy patients following surgery: a review. $J$ South Orthop Assoc. 2003; 12: 125-33.

2. Rattey TE, Leahey L, Hyndman J, Brown DC, Gross M. Recurrence after Achilles tendon lengthening in cerebral palsy. J Pediatr Orthop. 1993; 13: 184-7.

3. Dietz FR, Albright JC, Dolan L. Medium term follow-up of Achilles tendon lengthening in the treatment of ankle equinus in cerebral palsy. Iowa Orthop J. 2006; 26: 27-32.

4. Boireau P, Laville JM. Percutaneous lengthening of the Achilles tendon in children with cerebral palsy. Technique and results. Rev Chir Orthop Reparatrice Appar Mot. 2002; 88: 705-9.

5. Greene WB. Cerebral palsy. Evaluation and management of equinus and equinovarus deformities. Foot Ankle Clin. 2000; 5: 265-80. 